\title{
Effect of Wound Position, Auxin, and Agrobacterium vitis Strain F2/5 on Wound Healing and Crown Gall in Grapevine
}

\author{
J. E. Creasap, C. L. Reid, M. C. Goffinet, R. Aloni, C. Ullrich, and T. J. Burr
}

First, second, and sixth authors: Department of Plant Pathology, and third author: Department of Horticultural Sciences, Cornell University, NYSAES, Geneva, NY 14456; fourth author: Department of Life Sciences, Tel Aviv University, Tel Aviv 69978, Israel; and fifth author: Institut für Botanik, Technische Universität Darmstadt, Schnittspahnstr. 3, D-64287 Darmstadt, Germany. Accepted for publication 7 December 2004.

\begin{abstract}
Creasap, J. E., Reid, C. L., Goffinet, M. C., Aloni, R., Ullrich, C., and Burr, T. J. 2005. Effect of wound position, auxin, and Agrobacterium vitis strain F2/5 on wound healing and crown gall in grapevine. Phytopathology 95:362-367.

Agrobacterium vitis is the causal agent of crown gall disease in grapevine, which can be severe in many regions worldwide. Vitis vinifera cultivars are highly susceptible to freeze injury, providing the wounds necessary for infection by $A$. vitis. Wound position in relation to the uppermost bud of cuttings was determined to be important in tumor development. Inoculated wounds below buds developed tumors, whereas

wounds opposite the bud did not, implying that indole-3-aectic acid flow contributes to tumor formation. If auxin was applied to wounds prior to inoculation with a tumorigenic A. vitis strain, all sites of inoculation developed tumors, accompanied by an increased amount of callus in the cambium. Wounds inoculated with an A. vitis biological control strain F2/5 prior to application of the pathogen did not develop galls. A closer examination of these wounds determined that callus cells formed in the cambium during wound healing are susceptible to transformation by the pathogen. Although the mechanism by which F2/5 prevents transformation is unknown, our observations suggest that F2/5 inhibits normal wound healing by inducing necrosis in the cambium.
\end{abstract}

Agrobacterium vitis causes crown gall disease on trunks and canes of grapevines, often resulting in reduced growth and, potentially, the death of vines (6). The disease occurs in grapevinegrowing regions throughout the world and is especially significant in climates where cold winter temperatures cause wounds that are essential for initiation of infection. Most cultivars of Vitis vinifera L. are highly susceptible to freeze injury and crown gall infections $(18,25)$. Disease incidence can be very high in vineyards, and there are currently few strategies for disease management. The A. vitis infection process is thought to be similar to that of A. tumefaciens $(5,6,28)$, because $A$. vitis also requires a wound for infection and carries similar Ti plasmids, by which the strains are categorized (17).

Tumorigenic strains and nontumorigenic strains of $A$. vitis have been isolated from grapevines (9), and all induce a necrosis that is specific to grape roots and shoot explants $(7,23)$. The significance of the necrosis on grape roots is unknown. However, certain nontumorigenic strains, such as F2/5, induce a relatively high level of necrosis and also inhibit crown gall on grapevines when the strains are applied to wounds prior to inoculation with tumorigenic strains $(4,8,9,21)$. Although the mechanism of this grapevine-specific biological control is unclear, it does not appear to be associated with antibiotic production or with competition for attachment to host cells $(4,10)$.

Freeze injuries to vines occur throughout the winter; however, crown gall development is not observed until early summer. The timing of tumor formation coincides with the period during which

Corresponding author: J. E. Creasap; E-mail address: jec53@cornell.edu

* The $\boldsymbol{e}$-Xtra logo stands for "electronic extra" and indicates that the online version contains supplemental material not included in the print edition. Figure 2 is in color online.

DOI: 10.1094/PHYTO-95-0362

(C) 2005 The American Phytopathological Society renewed auxin flow within the vine stimulates reactivation of the vascular cambium (M. C. Goffinet, unpublished data) and the degradation of dormancy callose $(2,3)$. After freeze injury occurs in the winter, this renewed auxin flow is generated by one or more sturdy shoots, the growth of which is necessary for cell division to occur in the cambium during springtime growth (18). Basal transport of auxin from shoots to wounds is known to play an essential role in wound healing (1) and also has been correlated with crown gall development $(11,19,22)$. Pre-culture of Arabidopsis thaliana genotypes with phytohormones-such as auxin and cytokinins-increased competency for infection by Agrobacterium tumefaciens $(11,19)$. These studies identified dedifferentiating cells associated with the cambium as those most susceptible to transformation by A. tumefaciens (19) and showed that auxin can increase susceptibility of cells to transformation (11). Other studies have indicated that auxin accumulation at basal wounds of grape shoot explants is related to crown gall infection by A. vitis (22).

During the winter, callose, a $\beta$-1,3-glucan, plugs phloem sieve tubes and helps to protect vines from cold temperatures (13). Renewed auxin flow in the spring leads to the degradation of dormancy callose in grapevines, which was found to be nearly complete by late spring to early summer in the cold climate viticultural region of Ontario, Canada (3). Callose also is a defense barrier against pathogens, prevents disease development, and is known to accumulate near wounds, becoming a boundary between damaged tissue and healing tissue (24).

The goals of this study were to determine how the tumorigenic A. vitis strain K306 and the nontumorigenic strain F2/5 affect wound healing in woody grapevine tissues and to determine the effect of auxin and callose on crown gall development.

\section{MATERIALS AND METHODS}

Bacterial strains. A. vitis strains were grown on potato dextrose agar (PDA) (Difco Laboratories, Detroit), or PDA amended 
with kanamycin $(50 \mu \mathrm{g} / \mathrm{ml})$. Escherichia coli strain S17-1 (p35SGUSINT) was grown on Luria-Bertani (LB) medium with kanamycin $(50 \mu \mathrm{g} / \mathrm{ml})$ at $28^{\circ} \mathrm{C}$. To visualize cells or tissues transformed by $A$. vitis, we constructed the tumorigenic A. vitis strain K306 with a plasmid (p35SGUSINT) encoding $\beta$-glucuronidase (GUS) (26). A. vitis strain K306 carrying p35SGUSINT was generated through conjugation with S17-1 (p35SGUSINT) (23, 26). This construct contains an intron that assures expression of the uidA (GUS) gene only in eukaryotic cells, as well as T-DNA border regions for Vir protein recognition and transfer. Therefore, during infection of plant cells, K306 (p35SGUSINT) may transfer T-DNA regions from pTiK306, from the p35SGUSINT plasmid, or from both. Plant cells transformed with p35SGUSINT will express uidA, which encodes GUS. When GUS cleaves its substrate, 5-bromo-4-chloro-3-indolyl- $\beta$-D-glucuronic acid (Xgluc), one of the products, dibromo-dichloroindigo, is an insoluble blue dye that is detectable in transformed cells (15).

Effect of wound position on infection. Results from previous experiments showed a high level of variability in gall development on rooted grape cuttings (unpublished data). This variability appeared to be related to the position of the inoculation site relative to the bud or growing shoot. To verify this phenomenon, dormant $V$. vinifera cv. Chardonnay cuttings were inoculated with strain K306 at wounds made either 1 to $2 \mathrm{~cm}$ directly below the uppermost bud or below the bud but on the opposite side of the cutting. Three wounds spaced 0.5 to $2.5 \mathrm{~cm}$ apart on the same side of the cutting, either below the bud or opposite to the side of the bud, were made with an electric drill to the depth of the pith using a 2.8-mm-diameter drill bit. A K306 water suspension $(50 \mu \mathrm{l})$ at $10^{8} \mathrm{CFU} / \mathrm{ml}$ (optical density at $600 \mathrm{~nm}=0.1$ ) was applied to each wound. The effect of F2/5 on crown gall development was tested by inoculating wounds with the same cell density of an F2/5 water suspension $24 \mathrm{~h}$ prior to inoculation with K306. Subsequently, the effect of auxin on crown gall was measured by applying a solution of indole-3-acetic acid (IAA; $5 \mu \mathrm{g} / \mathrm{ml}$ ) (Sigma-Aldrich, St. Louis) to wounds below and to wounds opposite the bud prior to treatment with F2/5 and/or K306. Sterile distilled water (SDW) replaced IAA, F2/5, or K306 in controls. Following treatment, the wounds were wrapped with Parafilm (Pechiney Plastic Packaging, Menasha, WI); the cuttings were planted in perlite and kept in a greenhouse with daytime temperatures ranging from 18 to $23^{\circ} \mathrm{C}$, and nighttime temperatures ranging from 15 to $21^{\circ} \mathrm{C}$. Gall diameter was measured at its widest point at 60 days postinoculation. Three to five cuttings were inoculated at the different wound positions per treatment, and the experiment was repeated three times.

Identification of cells susceptible to transformation and the effect of F2/5 on transformation and wound callus. For the following experiments three wounds approximately 1 to $2 \mathrm{~cm}$ apart and $3 \mathrm{~mm}$ deep were made perpendicular to the axis of the cane by tapping a razor blade into dormant Chardonnay cuttings. This method of wounding was used because it facilitated subsequent sectioning and observation of grape anatomy at the wound. All wounds were made on the bud side of the cutting beneath the uppermost bud. An F2/5 water suspension $\left(30 \mu \mathrm{l} ; 10^{8} \mathrm{CFU} / \mathrm{ml}\right)$ or SDW were applied to wounds $24 \mathrm{~h}$ prior to application of the same number of CFU of K306(p35SGUSINT). At 14, 21, and 28 days postinoculation, $200-\mu \mathrm{m}$ thick sections of wounds were incubated at $37^{\circ} \mathrm{C}$ for $\approx 24 \mathrm{~h}$ in a solution containing X-gluc ( $1 \mathrm{mg} / \mathrm{ml}$ dissolved in $N, N$-dimethylformamide), $5 \mathrm{mM}$ potassium ferrocyanide, $5 \mathrm{mM}$ potassium ferricyanide, and $0.1 \mathrm{M}$ phosphate buffer (17). In all, 10 sections were made from the top surface of the wound and 10 from the bottom surface. The location of GUSstained sites (i.e., transformed cells) was noted. Photographs were taken on a Zeiss Stemi 200-C stereomicroscope (Zeiss, Göttingen, Germany) with a Zeiss MC-80 microscope camera. The experiment was repeated four times, and three wounds from one cutting were sectioned on each observation day.
Effect of auxin on transformation and wound callus formation. Three wounds per cutting were made with razor blades as described above. Different concentrations of IAA (30 $\mu \mathrm{l}$ each) were added to wounds $\approx 2 \mathrm{~h}$ prior to application of K306(p35SGUSINT), and SDW replaced either IAA or K306(p35SGUSINT) in the controls. Four concentrations (1, 3, 5, and $7 \mu \mathrm{g} / \mathrm{ml}$ ) of IAA were tested, each one diluted from a stock solution of IAA in SDW at $100 \mu \mathrm{g} / \mathrm{ml}$. Each of the three wounds from one cutting from each of the four treatments and the controls was sectioned with a razor blade and tested for GUS activity. The stained sites indicative of GUS activity were counted, and the relative amount of cambium-associated callus was measured radially at the widest point in each section. Both measurements were assigned rating numbers based on the following scales. For transformation, $0=$ no transformed cells, $1=1$ to 3 transformed cell groups, $2=4$ to 7 transformed cell groups, $3=8$ to 12 transformed cell groups, and $4=13+$ transformed cell groups. For callus production, $0=$ no callus, $1=10$ to $44 \mu \mathrm{m}$ of callus, $2=45$ to $88 \mu \mathrm{m}$ of callus, $3=89$ to $130 \mu \mathrm{m}$ of callus, $4=131$ to $180 \mu \mathrm{m}$ of callus, and $5=>181 \mu \mathrm{m}$ of callus. Digital images were taken on an Olympus BX60 compound light microscope and camera (Olympus, Tokyo).

Effect of K306(p35SGUSINT) and F2/5 on callose degradation and wound healing in Riesling. Wounds were made on dormant $V$. vinifera cv. Riesling cuttings with razor blades as described above and inoculated with $30 \mu$ of SDW or with a bacterial suspension of either K306(p35SGUSINT) or F2/5 $\left(10^{8} \mathrm{CFU} / \mathrm{ml}\right)$. At 7,14 , and 36 days postinoculation, 2-cm-long cane sections containing the wound were bisected longitudinally; the wounded and nonwounded halves were kept in separate containers of $70 \%$ ethanol to prevent further callose formation or degradation. Wound-associated tissues then were sectioned with either a sliding microtome or razor blade into 75 - to $200-\mu \mathrm{m}$-thick sections. Sections were placed in $0.1 \%$ aniline blue $(5 \mathrm{mg} / \mathrm{ml}$ in glycerol) for $15 \mathrm{~min}$, rinsed with double-distilled water, and viewed under UV light of an Aristoplan epifluorescence microscope (filter block A: excitation BP 340 to $380 \mathrm{~nm}$; emission LP $430 \mathrm{~nm}$; Leica, Bensheim, Germany) to observe callose deposits in phloem sieve tubes. Micrographs were digitally reproduced from color slides taken with an Orthomat E camera system (Leica) on Kodak Ektachrome Elite 200 ASA daylight film.

\section{RESULTS}

Effect of wound position on infection. Callus developed in wounds inoculated with SDW, IAA, and A. vitis strain F2/5 without the addition of the tumorigenic A. vitis strain K306. The average gall or callus rating in these treatments was $\approx 0.5$ (Fig. 1); consequently, an average of 0.5 or lower was attributed to wound callus formation, not to crown gall development. Crown gall readily formed at the K306-inoculated wounds made below buds of cuttings (Fig. 1, open bars), but was suppressed at wounds on the opposite side of cuttings (Fig. 1, hatched bars). The addition of IAA at $5 \mu \mathrm{g} / \mathrm{ml}$ to the wounds on the bud side of the cutting did not affect gall size. However, when IAA was applied to wounds opposite the buds, all sites inoculated with K306 alone formed galls, and the average gall rating was greater than without IAA (Fig. 1). In wounds treated with F2/5, no galls formed, as observed previously $(3,6)$, and less callus developed than in water controls. The addition of IAA prior to application of F2/5 did not significantly increase callus development compared with F2/5 alone (Fig. 1).

Identification of cells susceptible to transformation and the effect of F2/5 on transformation. Wounds inoculated with K306(p35SGUSINT) showed high numbers of GUS-stained sites in undifferentiated callus cells associated with the cambium. The GUS sites were most readily observed at 14 to 21 days after inoculation (Fig. 2A). The transformed areas consistently ap- 
peared in the callus close to the phloem in the new secondary vascular tissues. Treatment of wounds with F2/5 prior to K306(p35SGUSINT) resulted in the absence of detectable GUS expression and the development of little to no callus at the cambium (Fig. 2B). Treating wounds with F2/5 alone also led to greatly reduced cambium-associated callus development. In wounds from vines inoculated after shoot development, application of F2/5 resulted in necrosis in the cambium after development of new vascular tissues (Fig. 2D). Wounded tissue in Chardonnay cane cuttings inoculated with SDW did not show this necrosis in the cambium (Fig. 2C). The same observations were made for each of the three wounds from each of the four replications of the experiment.

In a few of the inoculated and uninoculated samples, GUS expression was observed in the epidermis and in paratracheal parenchyma cells surrounding vessel elements.

Effect of auxin on transformation and callus formation. The addition of IAA to wounds prior to K306(p35SGUSINT) resulted in increased GUS expression; the highest activity occurred with IAA at $3 \mu \mathrm{g} / \mathrm{ml}$ (Fig. 3). The amount of cambium-associated wound callus also increased with increasing IAA concentrations, with or without inoculation with K306 (Fig. 3). However, at any given concentration, more callus formed in the presence of K306. When this wound callus formed, it developed above the wound site and grew down toward the bottom of the wound. Inoculation with K306 alone resulted in abundant callus formation, presumably due to the initiation of crown gall disease (Figs. 2A and 3). When IAA and K306 were added to wounds at the same time, greater amounts of cambium-associated callus formed than with either treatment alone (Fig. 3, squares).

Effect of K306(p35SGUSINT) and F2/5 on callose degradation and wound healing. The maximum amount of callose degradation and best wound healing were most clearly seen at 36 days postinoculation. There were no apparent differences in the relative amounts of callose present in wounds treated with water compared with those inoculated with K306(p35SGUSINT) or F2/5 (Fig. 2E and F). The observed progression of callose degradation in unwounded tissues was similar to that previously described; callose degradation proceeded in a centrifugal direction from the cambium $(2,13)$.

Following wounding, the phloem sieve tubes regenerated laterally and downward around the wound and reconnected to phloem sieve tubes below the wound (data not shown). In these sections,

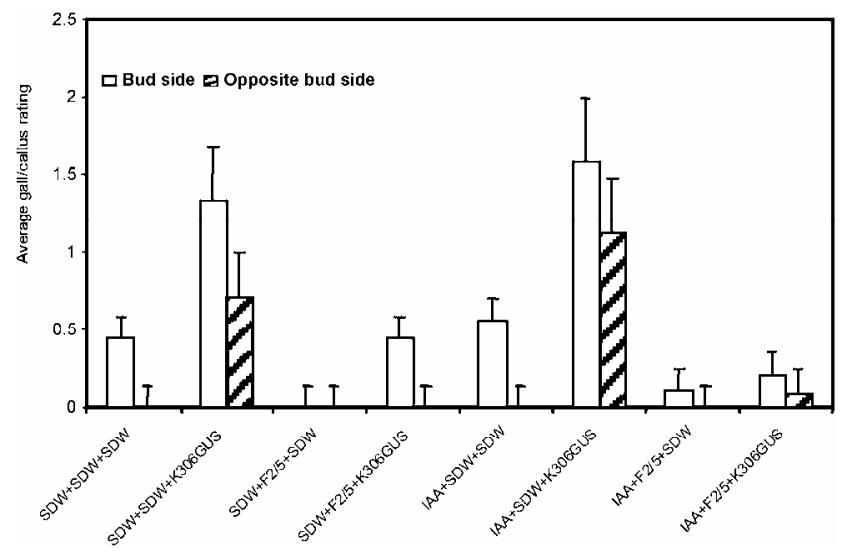

Fig. 1. Average callus rating in wounds made below the uppermost buds of dormant Chardonnay cuttings. Open bars represent inoculations made directly below the uppermost bud, and hatched bars represent inoculations made on the side of the cutting opposite the uppermost bud. An average callus rating of 0.5 relates to general wound callus formation, not crown gall development. Error lines represent standard error for each treatment. Gall diameters were measured at the widest sector and given a rating number based on the following scale: $0=$ no callus or gall, $1=<0.5 \mathrm{~mm}, 2=0.5$ to $0.99 \mathrm{~cm}$; and $3=\geq 1.00 \mathrm{~cm}$. radial regeneration around the wound was observed as bright, callose-covered sieve plates that formed parallel to the cambium. Callose distribution was irregular in wounds, in that middle sieve tube groups had no callose, whereas groups next to the cambium and close to the phellogen remained partially plugged with callose (Fig. 2E and F).

During infection with K306(p35SGUSINT), a similar callose distribution pattern was observed, and large quantities of disorganized wound callus formed in the cambium compared with the SDW treatments (Fig. 2E). Wounds treated with F2/5 exhibited similar callose distribution, and little wound callus was observed. In addition, conspicuous necrosis was observed at the cambium of F2/5-treated sections (Fig. 2F). Small, isolated areas of cambium-associated necrosis also were apparent following inoculation of K306(p35SGUSINT), but the necrosis seen in the F2/5-treated wounds generally covered the entire cambium.

\section{DISCUSSION}

It has been consistently observed that grape crown gall is most severe following winters when freezing temperatures cause injuries to trunks and canes of vines (6). Wounding is known to be essential for crown gall infection due to wound-released chemical signals that induce the expression of virulence genes in Agrobacterium spp. leading to the transfer of T-DNA to the plant cell $(5,28)$. Wounds also can secrete nutrients that facilitate chemotaxis and subsequent attachment of the bacteria to plant cells. In this article, we show that, during the process of wound healing, activation of the cambium generates cells that are susceptible to crown gall infection. Basipetal transport of auxin from buds to plant wounds, as well as the movement of cytokinins through plants, play essential roles in wound healing and regeneration of vascular tissues $(1,24)$. Auxin stimulates cambial activity resulting in the development of wound callus and subsequent differentiation of cells into new vasculature (24). We present several lines of evidence to indicate that auxin transport to the wound sites on woody grape cuttings stimulates vascular cambium activity, resulting in the generation of cells that are susceptible to transformation by $A$. vitis and development of crown gall. Wounds made opposite the buds, where less auxin transport to the wound is likely to occur, do not develop crown gall without the addition of exogenous auxin. Auxin application also stimulated an increase in cambium-associated callus and an increased number of GUS sites following inoculation with K306(p35SGUSINT). Other supporting evidence includes experiments done with cuttings that unknowingly had dead buds, in which case there presumably would be no auxin transport to wounds. In these experiments, no crown gall developed at any of the inoculation sites (data not shown).

Our results are consistent with previous research done with A. vitis on grape shoot explants and with other Agrobacterium spp.-plant systems. Stover et al. (22) reported that greater levels of transformation of grape shoots occurred when basal ends of explants were inoculated compared with apical ends, again suggesting that the basipetal transport of auxin enhanced the ability of $A$. vitis to infect cells in grape tissue. Sangwan et al. (20), also using a GUS reporter system, demonstrated that GUS expression occurred at wounds on Arabidopsis thaliana and that de-differentiating cells in the wounds were most susceptible to transformation. Furthermore, Chateau et al. (11) demonstrated that Arabidopsis genotypes that are resistant to transformation by Agrobacterium tumefaciens could be made susceptible via pretreatment of wounds with the phytohormones auxin and cytokinin. Therefore, auxin transport to wounds plays an important role in generation of cells that are susceptible to transformation by Agrobacterium spp. Our research indicates that A. vitis compromises the normal wound-healing process in woody grape tissue by infecting cells that, in the absence of the pathogen, differentiate into functional plant tissues. 
Following inoculation with K306(p35GUSINT), we observed abundant cambium-associated callus development but relatively few GUS sites. This may result because, as indicated previously, only a small proportion of transformed cells express the uidA gene, or because expression of uidA is variable depending on the developmental phase of the cell (15). Additionally, Rezmer et al. (19) noted that GUS activity in tumors in inoculated Ricinus plants was low and did not account for tumor size. Combining polymerase chain reaction (PCR) with reverse-transcription PCR, they showed that almost $100 \%$ of the nucleated tumor cells were transformed by $A$. tumefaciens carrying p35SGUSINT, but only $25 \%$ of these cells actually expressed the uidA gene.

We also observed that paratracheal parenchyma cells in the grape sections inoculated with K306(p35SGUSINT) and in water- inoculated controls occasionally stained light blue. Assays run at pH 4 and pH 7 did not reveal any endogenous GUS activity in grape; thus, it is unlikely these cells were producing any enzyme (data not shown). The paratracheal parenchyma cells which are involved in xylem loading also divide and differentiate during wound healing (12); therefore, they may be susceptible to Agrobacterium spp. infection. Because GUS expression was observed in the water controls, it also is likely that GUS-generating endophytes are present in the grape cuttings, as noted formerly by Stover et al. (22).

Callose deposition in the phloem of woody grape tissue has been studied in relation to seasonal vine development $(2,3)$, but not in response to wounding or pathogen invasion. For example, the accumulation of callose in Triticum aestivum L. confers
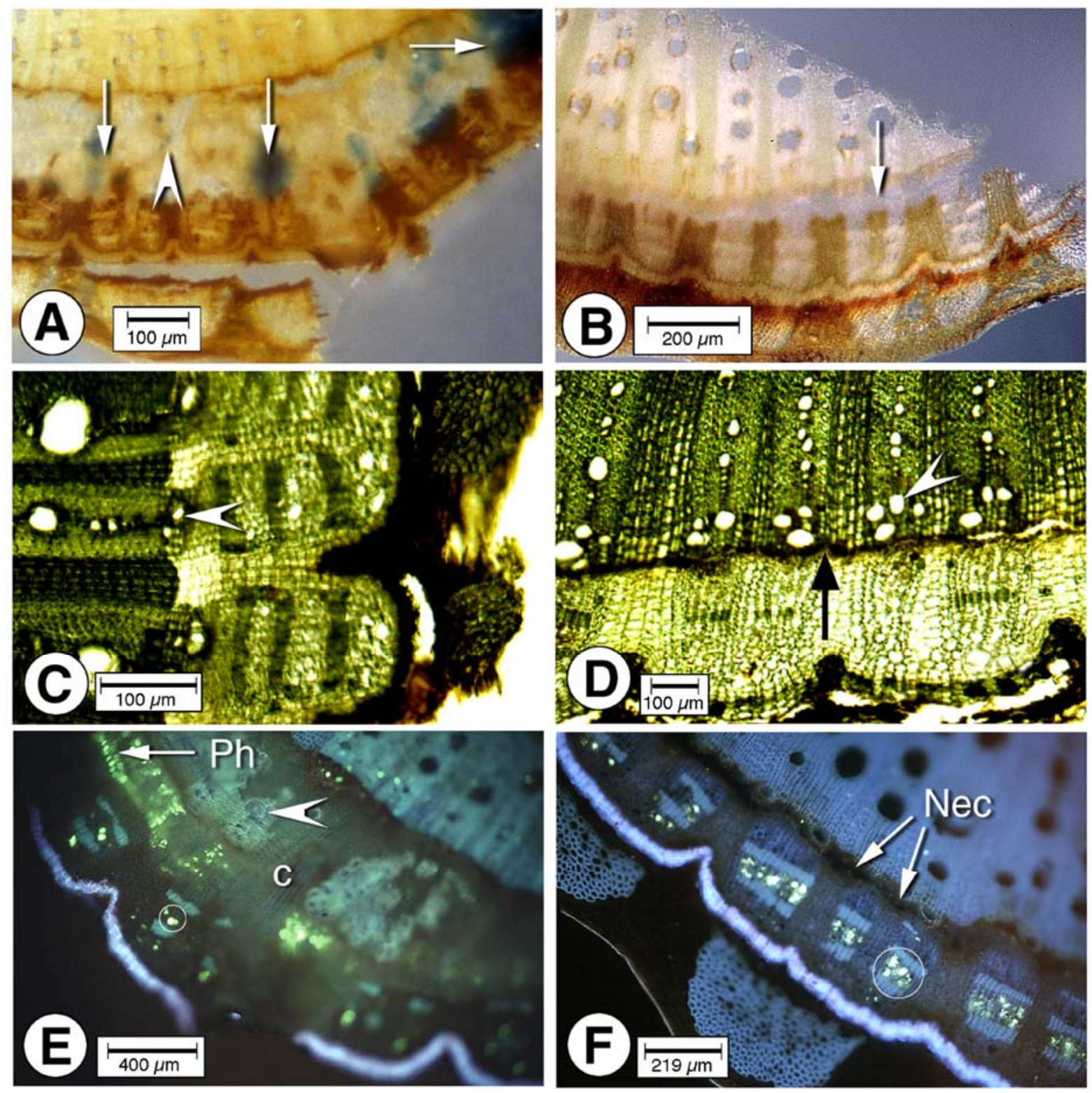

Fig. 2. A, Vitis vinifera cv. Chardonnay. Grape section from wound inoculated with Agrobacterium vitis strain K306 carrying p35SGUSINT. Disorganized callus (arrowhead) forms at the cambium. Blue, $\beta$-glucuronidase (GUS)-stained sectors (arrows) designate transformed cells or groups of cells. B, $V$. vinifera cv. Chardonnay. Grape section from wound inoculated with A. vitis biological control strain F2/5, followed $24 \mathrm{~h}$ later with strain K306 carrying p35SGUSINT. Little to no callus (arrow) forms at the cambium of the sections, and no transformed cells appear. C, V. vinifera cv. Chardonnay. Grape section showing normal wound healing in sterile distilled water control. Development of new xylem occurred (arrowhead) prior to inoculation. D, V. vinifera cv. Chardonnay. Grape section from wound inoculated with A. vitis biological control strain F2/5 alone after shoot growth. New xylem (arrowhead) and necrosis (black arrow) can be seen. E, $V$. vinifera cv. Riesling. Aniline blue stain of a section from a wounded vine treated with strain K306 carrying p35SGUSINT. Newly formed phloem (Ph) and lignified cells (arrowhead) can be seen in disorganized callus (C). Circled are several phloem sieve tubes containing callose. Courtesy of the Ullrich lab, Germany. F, V. vinifera cv. Riesling. Aniline blue stain of a section from a wounded vine treated with the biological control strain F2/5. A general necrosis along the cambium appears as brown cells (Nec), and callose in phloem sieve tubes is circled. Courtesy of the Ullrich lab, Germany. 


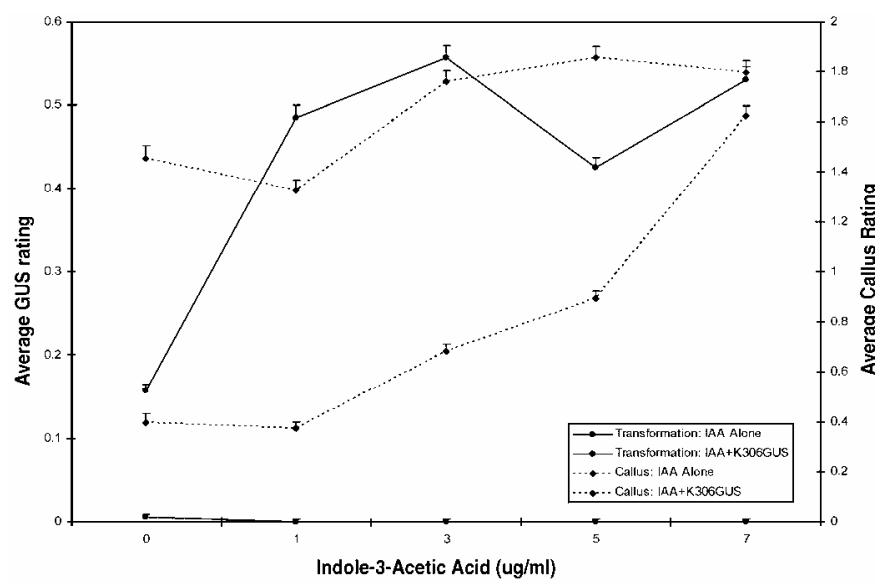

Fig. 3. Callus formation at the cambium (dashed lines) and transformation (solid lines) of cambium-associated callus cells in response to Agrobacterium vitis or varying concentrations of indole-3-acetic acid (IAA) from all sections made 14, 21, and 28 days postinoculation. Number of $\beta$-glucuronidase (GUS)stained cell groups were counted and rated with the following scale: $0=$ no transformed cells, $1=1$ to 3 transformed cells groups, $2=4$ to 7 transformed cell groups, $3=8$ to 12 transformed cell groups, and $4=13+$ transformed cell groups. Callus was measured at its widest point with a dissecting microscope at $\times 20$ and assigned to a rating scale where $0=$ no callus, $1=10$ to $44 \mu \mathrm{m}, 2=$ 45 to $88 \mu \mathrm{m}, 3=89$ to $130 \mu \mathrm{m}, 4=131$ to $180 \mu \mathrm{m}$, and $5=>181 \mu \mathrm{m}$. Error bars represent standard error for each treatment.

resistance to the wheat rust pathogen, Puccinia graminis (25), as well as in other resistant wheat cultivars (16). In our observations, we did not observe differences in the relative amount of callose accumulation in wounds treated with F2/5, K306, or water. Therefore, it appears that, unlike some other pathogens, A. vitis does not induce increased callose deposition. Further research is needed to determine if this response is host or pathogen related.

Although the mechanism of the biological control of crown gall by F2/5 is unknown, F2/5 prevents transformation of grape tissue when it is applied to wounds prior to inoculation with pathogenic strains of $A$. vitis $(4,8,10)$. The mechanism of control does not appear to be associated with antibiosis or with competition for attachment sites on the plant (10). In this article, we show that F2/5 prevents transformation of cells in wounds made to woody grape tissues. It also was observed that callus formation is reduced at wounds treated with F2/5, and no crown galls develop at the wounds over time. Most significant was the observation that, in wounds, F2/5 induces necrosis that was observed as a continuous dark band in the cambial zone. Although necrotic areas also were observed in K306-inoculated wounds, they were localized and not continuous like those inoculated with F2/5. This finding raises the question of whether biological control is related to the induction of cell death in the cambium by F2/5, thus preventing the development of wound callus cells that, as indicated above, are susceptible to crown gall infection. The induction of necrosis by strains of $A$. vitis previously has been reported as occurring on grape roots and on shoot explants (7). It was reported that the mechanism of necrosis appears to be related to the ability of A. vitis to cause a hypersensitive-like response (HR) on nonhost plants (14) and that it is regulated in a density-dependent manner (27). A luxR homolog in strain F2/5, aviR, has been identified (27) that is essential for induction of grape necrosis and the HR. We currently are testing necrosis and HR mutants of F2/5, including the aviR mutant, to determine their biological control phenotypes. In this way, it will be possible to determine whether root and cambial necrosis are mechanistically the same and whether the induction of necrosis is related to the biological control function of $\mathrm{F} 2 / 5$.

\section{ACKNOWLEDGMENTS}

This research was partially funded by NRI Competitive Grants Program/USDA award number 2002-35319-12582 and by the USDA Viticulture Consortium East Program and the NY Wine and Grape Foundation.

\section{LITERATURE CITED}

1. Aloni, R. 1995. The induction of vascular tissues by auxin and cytokinin. Pages 531-546 in: Plant Hormones: Physiology, Biochemistry and Molecular Biology. P. J. Davies, ed. Kluwer, Dordrecht, The Netherlands.

2. Aloni, R., and Peterson, C. 1991. Seasonal changes in callose levels and fluorescein translocation in the phloem of Vitis vinifera L. IAWA Bull. 12:223-234.

3. Aloni, R., Raviv, A., and Peterson, C. 1991. The role of auxin in the removal of dormancy callose and resumption of phloem activity in Vitis vinifera. Can. J. Bot. 69:1825-1832.

4. Bazzi, C., Alexandrova, M., Stefani, E., Anaclerio, F., and Burr, T. J. 1999. Biological control of Agrobacterium vitis using non-tumorigenic agrobacteria. Vitis 38:31-35.

5. Binns, A., and Thomashow, M. 1988. Cell Biology of Agrobacterium infection and transformation of plants. Annu. Rev. Microbiol. 42:575-606.

6. Burr, T., and Otten, L. 1999. Crown gall of grape: Biology and disease management. Annu. Rev. Phytopathol. 37:53-80.

7. Burr, T. J., Bishop, A. L., Katz, B. H., Blanchard, L. M., and Bazzi, C. 1987. A root-specific decay of grapevine caused by Agrobacterium tumefaciens and A. radiobacter biovar 3. Phytopathology 77:1424-1427.

8. Burr, T. J., and Reid, C. L. 1994. Biological control of grape crown gall with non-tumorigenic Agrobacterium vitis strain F2/5. Am. J. Enol. Vitic. 45:213-219.

9. Burr, T. J., Reid, C. L., Adams, C. E., and Momol, E. A. 1999. Characterization of Agrobacterium vitis strains isolated from feral Vitis riparia. Plant Dis. 83:102-107.

10. Burr, T. J., Reid, C. L., Tagliati, E., Bazzi, C., and Sule, S. 1997. Biological control of grape crown gall by strain F2/5 is not associated with agrocin production or competition for attachment sites on grape cells. Phytopathology 87:706-711.

11. Chateau, S., Sangwan, R., and Sangwan-Norreel, B. 2000. Competence of Arabidopsis thaliana genotypes and mutants for Agrobacterium tumefaciens-mediated gene transfer: Role of phytohormones. J. Exp. Bot. 51:1961-1968.

12. Cutter, E. 1971. Plant Anatomy: Experiment and Interpretation. Parts I and II. Addison-Wesley Publishing Company, Reading, MA.

13. Esau, K. 1948. Phloem structure in the grapevine, and its seasonal changes. Hilgardia 18:217-296.

14. Herlache, T., Zhang, H., Reid, C., Zheng, D., Basaran, P., Thaker, M., Burr, A., and Burr, T. 2001. Mutations that affect Agrobacterium vitisinduced grape necrosis also alter its ability to cause a hypersensitive response on tobacco. Phytopathology 91:966-972.

15. Jefferson, R., Kavanagh, T., and Bevan, M. 1987. GUS fusions: $\beta$ Glucuronidase as a sensitive and versatile gene fusion marker in higher plants. EMBO J. 6:3901-3907.

16. Kang, Z., Huang, L., and Buchenauer, H. 2002. Ultrastructural changes and localization of lignin and callose in compatible and incompatible interaction between wheat and Puccinia striiformis. Z. Pflanzenkrankh. Pflanzenschutz 109:25-37.

17. Otten, L., DeRuffray, P., Momol, E. A., Momol, M. T., and Burr, T. J. 1996. Phylogenetic relationships between Agrobacterium vitis isolates and their Ti plasmids. Mol. Plant-Microbe Interact. 9:782-786.

18. Pratt, C., and Pool, R. 1981. Anatomy of recovery of canes of Vitis vinifera L. from simulated freezing injury. Am. J. Enol. Vitic. 32:223-227.

19. Rezmer, C., Schlichting, R., Wächter, R., and Ullrich, C. 1999. Identification and localization of transformed cells in Agrobacterium tumefaciensinduced plant tumors. Planta 209:399-405.

20. Sangwan, R., Bourgeois, Y., Brown, S., Vasseur, G., and SangwanNorreel, B. 1992. Characterization of competent cells and early events of Agrobacterium-mediated genetic transformation in Arabidopsis thaliana. Planta 188:439-456.

21. Staphorst, J. L., van Zyl, F. G. H., Strijdom, B. W., and Groenewold, Z. E. 1985. Agrocin-producing pathogenic and nonpathogenic biotype-3 strains of Agrobacterium tumefaciens active against biotype-3 pathogens. Curr. Microbiol. 12:45-52.

22. Stover, E. W., Burr, T. J., and Swartz, H. J. 1996. Transformation of crown gall resistant and susceptible Vitis genotypes by Agrobacterium vitis. Vitis 35:29-33.

23. Stover, E., Swartz, H., and Burr, T. 1997. Crown gall formation in a diverse collection of Vitis genotypes inoculated with Agrobacterium vitis. Am. J. Enol. Vitic. 48:26-32. 
24. Taiz, L., and Zeiger, E. 2002. Pages 197, 432, 452 in: Plant Physiology, 3rd ed. Sinauer Associates, Inc., Sunderland, MA.

25. Tiburzy, R., and Reisener, H. J. 1990. Resistance of wheat to Puccinia graminis f. sp. Tritici association of the hypersensitive reaction with the cellular accumulation of lignin-like material and callose. Physiol. Mol. Plant Pathol. 36:109-112.

26. Vancanneyt, G., Schmidt, R., O'Connor-Sanchez, A., Willmitzer, L., and Rocha-Sosa, M. 1990. Construction of an intron-containing marker gene: Splicing of the intron in transgenic plants and its use in monitoring early events in Agrobacterium-mediated plant transformation. Mol. Gen. Genet. 220:245-250.

27. Zheng, D., Zhang, H., Carle, S., Hao, G., Holden, M. R., and Burr, T. J. 2003. A LuxR homolog, aviR, in Agrobacterium vitis is associated with induction of necrosis on grape and a hypersensitive response on tobacco. Mol. Plant-Microbe Interact. 16:650-658.

28. Zupan, J., Muth, T., Draper, O., and Zambryski, P. 2000. The transfer of DNA from Agrobacterium tumefaciens into plants: A feast of fundamental insights. Plant J. 23:11-28. 